\title{
ANALYSIS OF THE HEAD TAIL DAMPING AND GROWTH TIME FOR THE ESTIMATION OF THE ESRF MACHINE IMPEDANCE
}

\author{
P. Kernel, J.-L. Revol, R. Nagaoka, ESRF, Grenoble, France \\ G. Besnier, University of Rennes, France
}

\section{Abstract}

A theoretical and experimental characterisation of transverse damping mechanisms was initiated within the framework of the single bunch transverse instability studies. An attempt is made to estimate the machine impedance through the measurement of the head tail damping time as a function of the chromaticity. The presence of strong sextupole fields used for chromaticity compensation however induces large momentum and amplitude dependent tune shifts. A systematic study of the different damping mechanisms was therefore performed in order to quantify the different contributions, which for the ESRF storage ring are in the same range. Numerical simulations and record of the beam response to a kick were performed. The transverse signal decay after the excitation was observed on the tune monitor system used in zero span mode. The tune amplitude dependence which depends on the kick amplitude is discussed first. Then the modulation induced by chromaticity overcompensation which could blind the observation is described. The measurement of the head tail damping and growing time, under precise operating conditions, with positive and negative chromaticity, is presented. Finally, a model for the machine impedance is discussed.

\section{INTRODUCTION}

The optimisation of the machine operation and the understanding of the feedback system efficiency for future operation are the ultimate purpose of the transverse motion study at the ESRF. The modelling of the storage ring transverse impedance is a key point for the study of single bunch instability limitations. For this purpose, a campaign of measurements has been performed, their interpretation is under progress $[1,2]$. The head tail damping time and the growth rate of the transverse signal resulting from the interaction of the beam modes with the machine impedance are accessible physical quantities. These data are complementary in order to cross-check the impedance model resulting from measurements of mode merging observed at low current [2] and threshold curve associated to higher order head-tail instability [1] for the high current regime. Head tail damping time extraction from the measurement requires a methodical approach, which is part of this paper. The characterisation and the description of the different mechanisms occurring at ESRF, the measurement methods and data are presented. The results (in vertical plane) are compared to the tracking simulation in order to discuss the impedance model for single bunch.

\section{DIFFERENT MECHANISMS REVIEW}

The damping time measurement presents many difficulties in practice due to the fact that different other damping mechanisms can occur at the same time and spoil the observed signal.

\subsection{Transverse Radiation Damping}

Radiation damping mechanism presents a characteristic and constant decay time of $7 \mathrm{~ms}$ for the ESRF storage ring. This value is long enough not to perturb the head tail measurements.

\subsection{Chromatic Modulation}

After a transverse kick excitation, the frequency mixing of the individual particles oscillations, at slightly different betatron frequencies depending on the chromaticity $\xi=(d Q / Q) /(d p / p)$, results in a modulation of the transverse signal of period $T_{s}$ the synchrotron period [Fig.1.a and 1.b, $\left.T_{s}=0.5 \mathrm{~ms}\right]$. The peak widths, which only depend on the chromaticity, narrow when the chromaticity increases. To avoid confusing the chromatic modulation with the head-tail damping, the head tail damping decay should be much longer than $T_{s}$. In that case, from the amplitude of a few peaks, the envelope of the modulated signal can be extracted.
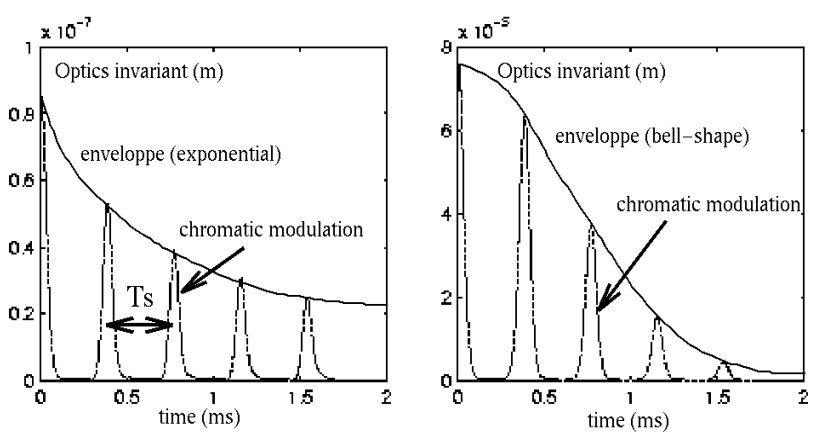

Fig 1.a (left) Modulated head tail damping (simul.) Fig 1.b (right) Modulated tune spread damping (simul.)

\subsection{Amplitude-Dependant Tune Spread Damping}

If the transverse kick is too large, additional tune spread is induced within the bunch by the non-linearity coming from the strong sextupoles fields. The corresponding damping signal resulting from this mechanism presents a bell-shape decay (Fig.1.b). The strength of the tune 
spread damping, which is independent of the beam current, increases with the kick amplitude, Fig.2. For the head tail damping time measurement, the kick should be chosen small to keep the tune spread damping time long enough to be negligible (in the order of a few $\mathrm{ms}$ ).

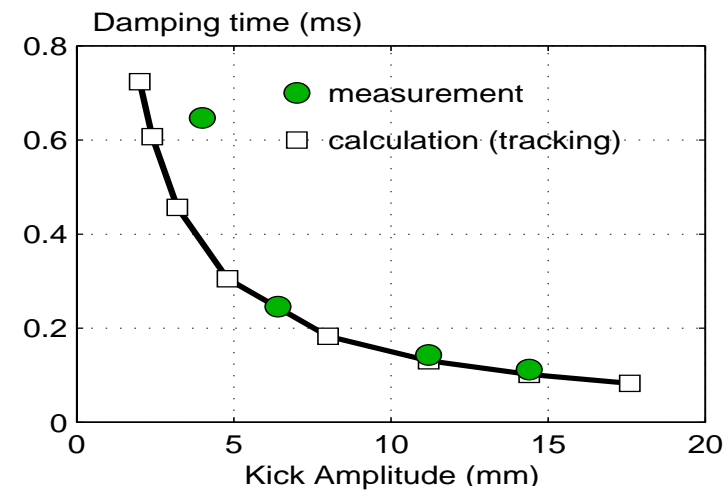

Fig 2: Amplitude-dependant tune spread damping time

\subsection{Head Tail Damping and Growing time.}

For Hermitian modes that are not interacting in a modecoupling regime, the head tail characteristic time for a mode $m$ is given by the formula (cf. [2]):

$$
\tau_{m}=\frac{2^{2 m} m !}{(2 m-1) ! !} \frac{\sigma_{\tau}}{I} \frac{4 \sqrt{\pi} E / e}{\beta} \frac{\sum_{p} h_{m}\left(\omega_{p}-\omega_{\xi}\right)}{\sum_{p} \operatorname{Re}(Z(\omega)) h_{m}\left(\omega_{p}-\omega_{\xi}\right)}
$$

$$
\text { with } \omega_{p}=\omega_{\beta}+m \omega_{s}+p \omega_{0} \text {. }
$$

The mode spectrum $h_{m}\left(\omega_{p}-\omega_{\xi}\right)$, is shifted in frequency by $\omega_{\xi}=\omega_{0} Q_{\beta} \xi / \alpha$, proportional to the chromaticity. The measurement of the head tail damping or growing time $\tau_{m}$ versus chromaticity, is characteristic of the interaction of the mode spectrum with the real part of the transverse impedance $Z(\omega)$. For the head tail mechanism, the transverse pick-up signal is exponential (fig.1a). The damping time $\tau_{m}$ should be inversely proportional to the peak current $I / \sigma_{\tau}$. and to the optical beta function $\beta$. These parameters can be mastered during the experiments. The bunch length which also defines the width of the mode spectrum $h_{m}\left(\omega_{p}-\omega_{\xi}\right)$, should be long in order to scan the impedance in more detail.

\section{HEAD TAIL DAMPING TIME $(\xi>0)$}

\subsection{Measurement Methods}

At positive chromaticities, the head tail damping time has been scanned versus $\xi$, in both vertical and horizontal planes, much below the current threshold. The transverse excitation is produced by a kick of the injection kicker for the horizontal plane, or by the gated tune monitor shaker for the vertical plane. The particle distribution resulting from the kick is equivalent to a low frequency shaker mode, and its corresponding envelope spectrum for Gaussian beam is (different of the spectrum of the Hermitian modes assumed in Eq.1 when $m \neq 0$ ):

$$
\begin{gathered}
h_{m}\left(\omega-\omega_{\xi}\right)=\exp \left[-\frac{1}{2}\left(\left(\omega-\omega_{\xi}\right)^{2}+\left(\omega_{s h}-\omega_{\xi}\right)^{2}\right) \sigma_{\tau}^{2}\right] \times \\
I_{m}\left[\left(\omega-\omega_{\xi}\right)\left(\omega_{s h}-\omega_{\xi}\right) \sigma_{\tau}^{2}\right]
\end{gathered}
$$

where $\mathrm{I}_{m}$ is the modified Bessel's function of the $1^{\text {st }}$ kind and $\omega_{s h} \approx 0$ is the shaker frequency, which is null for a kick. Kicked and shaked modes present exactly the same spectrum.

Synchronously with a kick, the transverse signal decay is measured with a spectrum analyser used in the zero span mode adjusted to the betatron frequency. This method improved by averaging was efficient for the detection of very low response defined by the following operating conditions:

- Low intensity per bunch, necessary for a head tail damping to be longer than $T_{s}=0.5 \mathrm{~ms}$, to be able to extract the envelope from the chromatic modulation.

- Small transverse kick to avoid the tune spread damping. The absence of tune spread can be check by the linear dependence with current.

- Increased average intensity, in some cases, to keep the transverse signal out of the noise level, (few bunch modes, with low single bunch intensity).

On Fig.3b, the expected shape of the head tail damping time after a kick has been computed with a tracking code, using a BBR model for the transverse vertical impedance with $f_{r}=22 \mathrm{GHz}, Q=1, R \times \beta=13.5 \mathrm{M} \Omega$.

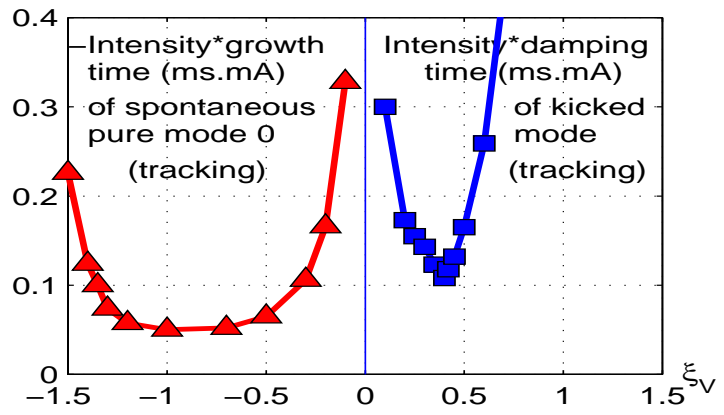

Fig3a(left) Head tail growth time simul. (mode 0)

Fig3b(right) Head tail damp. time simul. after a kick

\subsection{Results}

According to theory, the damping time should increase when $\xi$ is close to 0 , because of the cancellation due to the anti-symmetric real part of $Z(p)$; and increase at large $\xi$, due to the decreasing of impedance. The curve should present a minimum at the resonant frequency of the BBR model. These curves (Fig.4 right, vert. plane) do not present the expected shape and no proportionality with the current, which is a hint to say that the observed damping time was not a pure head tail phenomena. This discrepancy with the theory might come from a measurement problem. Despite the efforts employed to avoid all the perturbing phenomena and to extract the pure head tail damping mechanism, it seems that another mechanism has polluted the measurement. The obtained results should be clarified by further theoretical studies. 


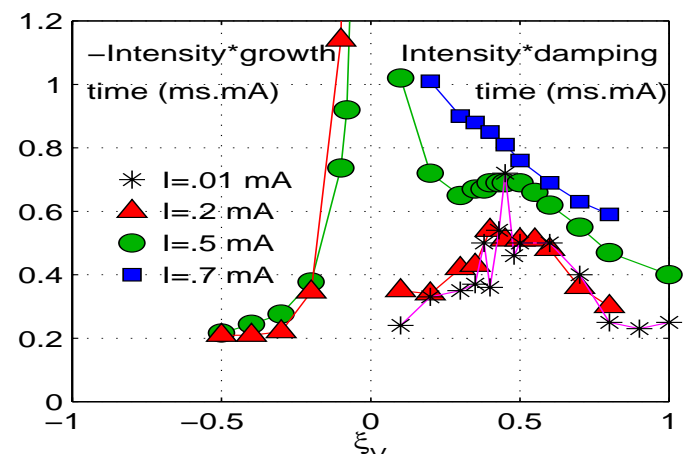

Fig 4: Measured head tail growth and damping times

\section{HEAD TAIL GROWING TIME $(\xi<0)$}

\subsection{Measurement Methods}

Growth rate measurement of the strongly unstable mode 0 has been realised in the negative chromaticity regime. In that case the spectrum, which is not distorted by any kick, is purely Gaussian. As the mode grows, the amplitude-dependent tune spread filamentates the beam and leads to a destruction of the initial mode coherence. A saw-tooth motion with a period in the range of millisecond may be observed at this stage (Fig.5), which is supposed to be due to the vanishing of the head-tail growth for a filamented beam. The radiation damping then concentrates the particles to the initial state. The strong tune spread damping would be a characteristic effect of the ESRF optics. It allows storing beam above head-tail threshold. For the measurement, the beam is stabilised with the transverse feedback system, which send the vertical transverse signal to the shaker, with proper gain and phase. At a given time, the feedback is suppressed for a few milliseconds and the transverse signal is analysed synchronously. (Fig.5 and 6)

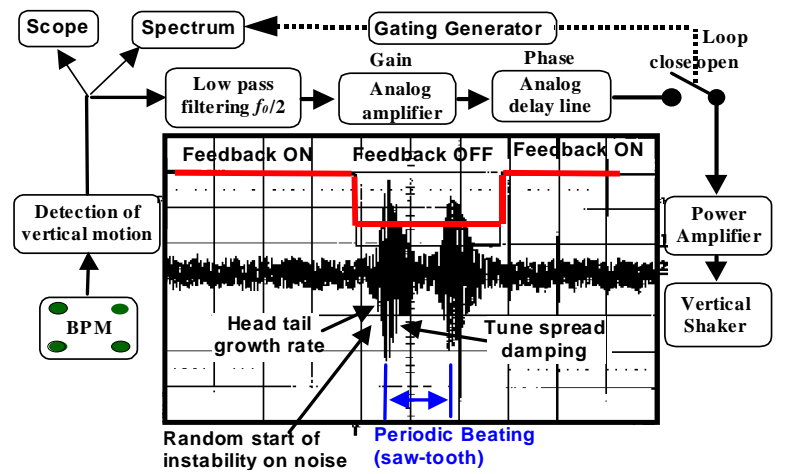

Fig 5: Measurement set-up at negative chromaticities

This method allows to go up to $\xi=-0.5$, but at larger negative vertical chromaticities, the feedback becomes inefficient. Due to the large mode spectrum shift by $\omega_{\xi}$, the measurement system which works at low frequency cannot pick-up the mode signal when $\xi$ is too far from 0 .

\subsection{Results}

The growing time which monotonously decreases up to $\xi=-0.5$, is in agreement with the $22 \mathrm{GHz}$ BBR impedance model given by other modelling methods [1] (Fig.4 left). Nevertheless, the limitation of the measurement at $\xi=-0.5$ (corresponding to $-13 \mathrm{GHz}$ ) does not allow to measure the turning point where the growing time should increase again. According to the theoretical curve, the turning point should be located at $\xi=-0.8$ (Fig.3.a). With a scaling of the parameter $R$, the measurement confirms that the $22 \mathrm{GHz}$ BBR is a reasonable model for the ESRF storage ring impedance. A possibility to measure the turning point could be to lengthen the spectrum by shortening the bunch. For a given $\xi$, this will increase the spectrum amplitude at zero frequency and improve the efficiency of the detected signal and of the feedback.

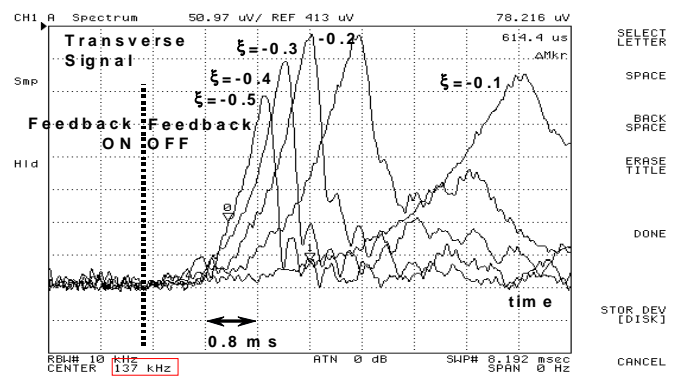

Fig 6: Growth rate as a function of the chromaticity

\section{CONCLUSION}

The different damping mechanisms at ESRF have been investigated and observed. The measurement of the head tail damping time cannot be interpreted but further theoretical studies will be done to understand the observation. The measurement of the growth time was successful and is compatible with BBR impedance model. Attempts are in progress to understand the imaginary part of the impedance by measuring the mode 0 detuning versus $\xi$, in the negative chromaticity regime.

\section{ACKNOWLEDGEMENTS}

The authors would like to thank E. Plouviez for his support for measurements and diagnostics, and N. Michel for the feedback development.

\section{REFERENCES}

[1] R. Nagaoka, J.-L. Revol, P. Kernel and G. Besnier, "Transverse Instabilities in the ESRF Storage Ring: Simulation, Experimental Results and Impedance Modelling”, PAC'99, New-York, 1999.

[2] J. Jacob, P. Kernel, R. Nagaoka, J.-L. Revol, A. Ropert, G. Besnier, "Experimental and Theoretical Studies of Transverse Single Bunch Instabilities at the ESRF”, EPAC'98, Stockholm, 1998.

[3] F.J. Sacherer, "Transverse Bunched-Beam Instabilities", CERN/PS/BR 76-21, Geneva, 1976. 